\title{
Frozen Section Evaluation in Head and Neck Oncosurgery: An Initial Experience in a Tertiary Cancer Center
}

\author{
Sangeetha K NAYANAR ${ }^{1}$ (D), Aswathi KRISHNAN M${ }^{1}$ (D), Mrudula K $I^{2} \stackrel{\mathbb{D}}{\mathbb{D}}$, Sajith Babu THAVAROOL $\mathrm{P}^{3}$ (D), \\ Shivakumar THIAGARAJAN ${ }^{4}$
}

\author{
'Department of Clinical Laboratory Services and Translational Research, Malabar Cancer Centre, THALASSERY, KERALA, INDIA \\ ${ }^{2}$ Department of Oral Pathology, Kannur Dental College, ANJARAKANDY, KERALA, INDIA \\ ${ }^{3}$ Department of Surgical Oncology, Malabar Cancer Centre, THALASSERY, KERALA, INDIA \\ ${ }^{4}$ Department of Head and Neck Surgical Oncology, Tata Memorial Centre, MUMBAI, INDIA
}

\begin{abstract}
Objective: Frozen section evaluation is routinely used by oncosurgeons across specialties for rapid assessment of the presence of tumor in any tissue and its most common use is in surgical margins. Today, the use of intraoperative frozen-section evaluation of surgical margins is an accepted and frequent practice in head and neck oncology. This study aims to determine the efficacy and accuracy of frozen sections in head and neck cancer patients and compare the results with the respective paraffin sections and also to analyze the reasons for any disparity between them.

Material and Method: A retrospective study was conducted to evaluate efficacy and accuracy of frozen section in head and neck cancer of 265 patients, treated at a tertiary cancer centre hospital between January 2013 to December 2014.

Results: Out of 265 cases, it was found that $12.6 \%$ of these sections showed true positivity, $6.3 \%$ false positivity, $2.9 \%$ false negativity and $78.2 \%$ true negativity. The study also shows a sensitivity of $82.05 \%$ and specificity of $96.46 \%$.

Conclusion: Our study shows that intraoperative frozen section reports are specific and highly sensitive. We recommend a minimum of 3-4 sections, optimum cryostat temperature, good section thickness and quality staining for a good concordance rate.
\end{abstract}

Key Words: Frozen sections, Margins of excision, Head and neck neoplasms

\section{INTRODUCTION}

Frozen section evaluation is a key technique used by pathologists during the intraoperative consultation (1). It plays an important role in the surgical management of patients with neoplastic disease and is an essential component for a surgical theatre complex. The modern frozen section as practiced today was developed by Dr. Louis B Wilson in 1905 (2). After the introduction of the cryostat in 1960, frozen section became a highly accurate procedure for assessing margin status intraoperatively (3). It is routinely used by surgeons across specialties for rapid assessment of presence of tumor in tissues, the most common being the surgical margins.

During oncosurgery, it is important for the surgeon to know if the surgical margin is clear of malignant cells $(2,4$, 5). The report given is usually binary in nature and it just indicates the presence or absence of tumor in the sample. Based on this report, the surgeon makes a decision on whether to make additional resections or not.

(Turk Patoloji Derg 2019, 35:46-51)

Received : 13.05.2018 Accepted : 13.08.2018
The use of intraoperative frozen section evaluation of surgical margins is an accepted and frequent practice in head and neck oncosurgery (6). Frozen section analysis is a multistep process, beginning with retrieval of the specimen from the patient by the surgeon followed by preparation of slides, their microscopic examination and possible specimen triage for further workup, and finally rendering the frozen section (FS) diagnosis. The frozen section diagnosis is usually communicated verbally to the surgeon by the pathologist via telephone, intercom, video conference (7), and sometimes in person via an intermediary (e.g., nurse or other OT personnel). Such reports enable the surgeon to decide on whether further resections are needed or not.

\section{MATERIAL and METHODS}

A retrospective study was conducted to evaluate efficacy and accuracy of a frozen section in head and neck cancer involving 265 patients after obtaining informed consent from the participants and the institutional review board of Malabar Cancer Centre. Data was collected from the

Correspondence: Aswathi KRISHNAN M

Department of Oncopathology, Malabar Cancer Centre,

Moozhikara PO, THALASSERY, KERALA, INDIA

E-mail: aswathikm99@gmail.comＰhone: +919061033644 
records of frozen section reports of head and neck cancer patients, MCC, during the period from 2013 January to 2014 December. All head and neck tumor resections which had intraoperative consultations involving frozen sections were considered. Patients with biopsy-proven head and neck carcinoma of all age groups with no sex predilection were included in the study. During the procedure the cryostat temperature was kept at $-22^{\circ} \mathrm{C}$ and sections were cut at 6 microns thickness with 2-3 sections per each block. The apparatus used was Leica CM 1850 UV. Details of the frozen section reports were collected along with age, gender and diagnosis. Frozen section reports were analyzed for tumor, surgical margins and lymph node metastasis. For all cases reported as positive margin, re-resections were done and all specimens were sent for routine margin analysis. Permanent section diagnosis is widely considered to be the datum in this diagnosis area (5). Reports were cross checked with the corresponding paraffin sections and analyzed for any mismatches. No patients received neoadjuvant chemotherapy prior to surgery.

Cases sent to our department for frozen section evaluation included those cases for primary diagnosis as well as cases for margin status and/or lymphnode metastasis. A total of 265 patients were selected and 734 sections were analyzed. After taking tissue bits for frozen sections, the remaining tissue was used for formalin fixation and for taking permanent sections. Frozen sections are critical to immediate surgical management $(6,8)$. Studies have also shown that survival rates are decreased if resection margin is found to be positive $(4,9)$. A routine review of frozen section can improve the accuracy of the procedure $(5,8)$.

Primary objective:

- Baseline the error rates of frozen section in our institution

Secondary objective:

- Recognize factors affecting the error rates

- Discuss improvements for the common factors causing the error

Statistical analysis was performed on the collected data using the statistical software SPSS. Crosstab analysis was used to find the sensitivity and specificity values. LR+ and LR- values were derived from sensitivity and specificity. The confidence intervals were obtained using the ROC curve analysis in MedCalc software, version 18 (MedCalc Software, Acacialaan 22, Belgium, https://www.medcalc. org/). ROC curve analysis figures were cross verified using the diagnostics test evaluation tool of MedCalc.

\section{RESULTS}

A total of 265 patients were selected and 734 sections were analyzed. The patient demographic data is summarized in Table I. Margin analysis was done for 195 cases, 41 cases were analysed for lymph node metastasis, and 45 cases were sent for primary tumor analysis. There are overlaps and each test was carried out on a need basis. The majority of the cases were squamous cell carcinoma (Figure 1A,B$2 \mathrm{~A}, \mathrm{~B})$. Other carcinomas like malignant tumors of thyroid carcinomas (Figure $3 \mathrm{~A}, \mathrm{~B}$ ) were also observed. There were 15 cases which showed disparity out of which seven cases were reported as false negative and eight cases were reported as false positive. The overall statistics are summarized in Table II.

Table I: Demographic details of cases.

\begin{tabular}{lccc}
\hline & Total (265) & Men (179) & Woman (86) \\
\hline Min. age & 29 & 29 & 32 \\
\hline Max. age & 83 & 83 & 82 \\
\hline Mean age & 59.45 & 58.41 & 61.61 \\
\hline Median age & 60 & 60 & 63.5 \\
\hline Std. Dev. age & 11.22 & 11.5 & 10.36 \\
\hline
\end{tabular}

Table II: Overview of the results.

\begin{tabular}{lcc}
\hline & No. of cases & \% \\
\hline True negatives & 218 & 82.26 \\
\hline True positives & 32 & 12.07 \\
\hline False positives & 8 & 3.02 \\
\hline False negatives & 7 & 2.64 \\
\hline Total cases & 265 & 100 \\
\hline
\end{tabular}

Table III: Distribution of the cases.

\begin{tabular}{lcc}
\hline Sites & No. of cases & $\%$ \\
\hline Tongue & 88 & 33.21 \\
\hline Buccal mucosa & 64 & 24.15 \\
\hline Floor of mouth & 15 & 5.66 \\
\hline Lower Alveolus & 23 & 8.68 \\
\hline Upper Alveolus & 13 & 4.91 \\
\hline Palate & 8 & 3.02 \\
\hline Retromolar trigone & 5 & 1.89 \\
\hline Lip & 6 & 2.26 \\
\hline Gingivo-buccal sulcus & 4 & 1.51 \\
\hline Pharynx & 5 & 1.89 \\
\hline Larynx & 16 & 6.04 \\
\hline Others & 18 & 6.79 \\
\hline
\end{tabular}


Table IV: Comparison of frozen section results with the permanent sections.

\begin{tabular}{lccc}
\hline Diagnosis & Total cases & False positives & False Negatives \\
\hline Carcinoma of tongue & 6 & 4 & 2 \\
\hline Carcinoma of buccal mucosa & 3 & 1 & 2 \\
\hline Carcinoma of alveolus & 3 & 2 & 1 \\
\hline Carcinoma of floor of the mouth & 1 & 0 & 1 \\
\hline Carcinoma of epiglottis & 1 & 1 & 0 \\
\hline Carcinoma of retromolar trigone & 1 & 0 & 1 \\
\hline
\end{tabular}

The prominent sites affected were the tongue and buccal mucosa. Table III has the details of the sites. Comparison of frozen section results with the permanent sections are shown in Table IV.

Table V shows observed values and interval estimate for sensitivity, specificity, disease prevalence and likelihood ratios along with $95 \%$ confidence interval for these values.

\section{DISCUSSION}

Frozen section analysis during intraoperative consultation is important in ensuring adequate margin clearance (5, 10, 11). Margin clearance is essential in head and neck oncosurgeries $(4,12)$ as it reduces the recurrence. During intraoperative consultations, the patient will still be under anesthesia and the average time taken for frozen section is about 20 minutes $(8,13)$ when done by only one seasoned pathologist.
Table V: Values and interval estimates.

\begin{tabular}{lcc}
\hline & Value & 95\% CI \\
\hline Sensitivity & $82.05 \%$ & $66.47 \%$ to $92.46 \%$ \\
\hline Specificity & $96.46 \%$ & $93.14 \%$ to $98.46 \%$ \\
\hline Disease prevalence & $14.72 \%$ & $10.68 \%$ to $19.57 \%$ \\
\hline +ve Likelihood Ratio & 23.18 & 11.55 to 46.5 \\
\hline -ve Likelihood Ratio & 0.19 & 0.10 to 0.36 \\
\hline
\end{tabular}

There were total of 15 discordant cases. However, only one of these discordant cases had an impact on immediate clinical management. We also observed that there was no discordance in the cases where a lymph node analysis was done. A study by White and Trotter (14) has shown very low rates for discordances in case of lymph node metastases (4 out of 427 cases), which matches our experience. Similarly, a study by Hatami et al. mentions only one discordant
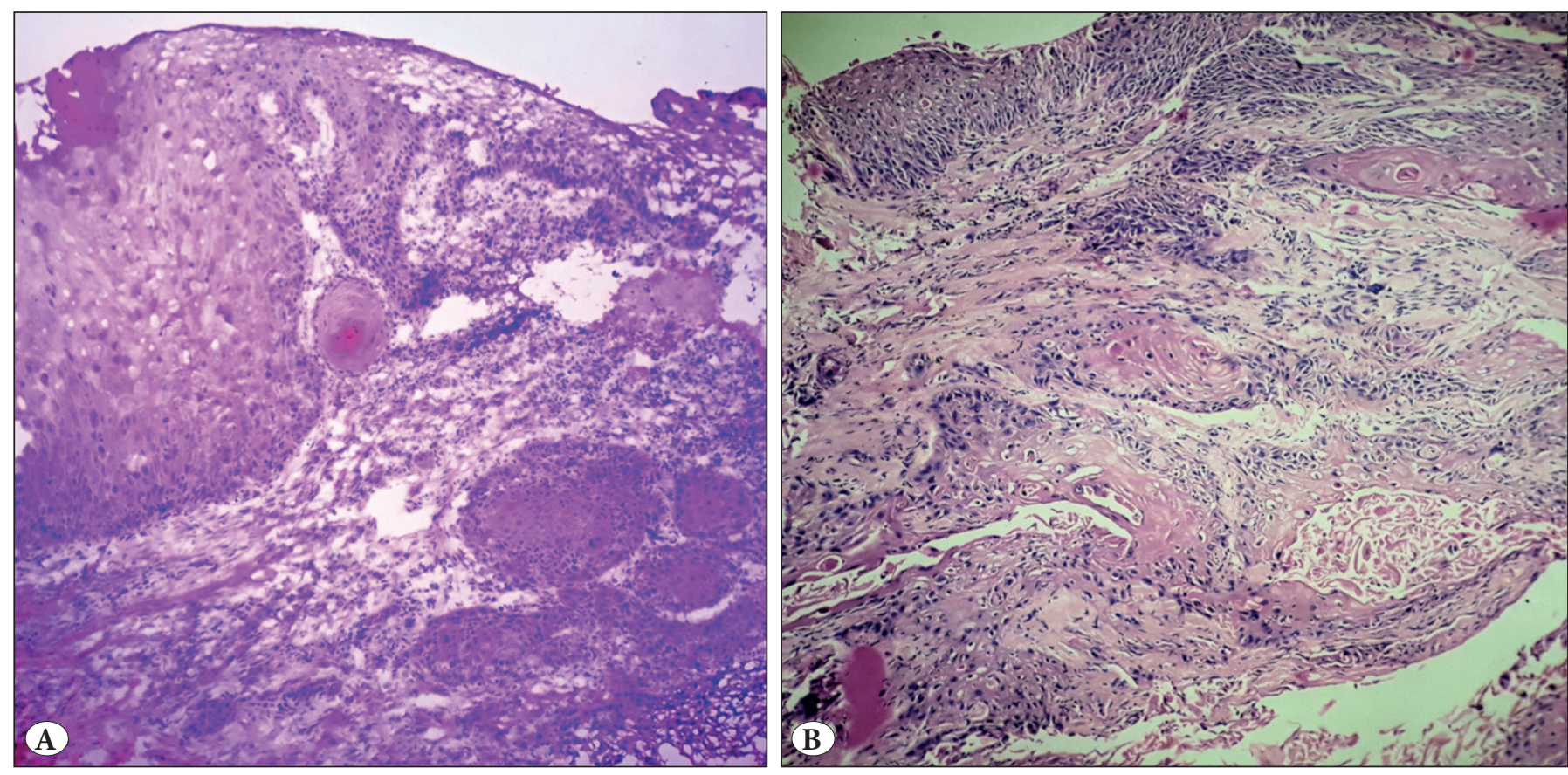

Figure 1: A) Frozen section of squamous cell carcinoma (H\&E; x100). B) Permanent section of squamous cell carcinoma (H\&E; x100). 
case out of 23 lymph nodes analysed (5). However, there are many other studies where discordance/deferral rates involving lymph node frozen sections are high $(6,8)$.

Analyzing the reasons for the disparity, it was noted that an inadequate number of deeper sections lead to false negativity in two cases of invasive carcinoma. Many studies have also pointed out similar reasons for false negatives $(6,8,10,15)$. Technical pitfalls such as thickness of the frozen section and folding were the reasons that lead to false negativity in the remaining five cases. Issues in staining by rapid $\mathrm{H} \& \mathrm{E}$ methods (e.g. cases of normal epithelium misinterpreted as mild/moderate dysplasia) lead to false positive cases. The details of the discordant cases are described below.
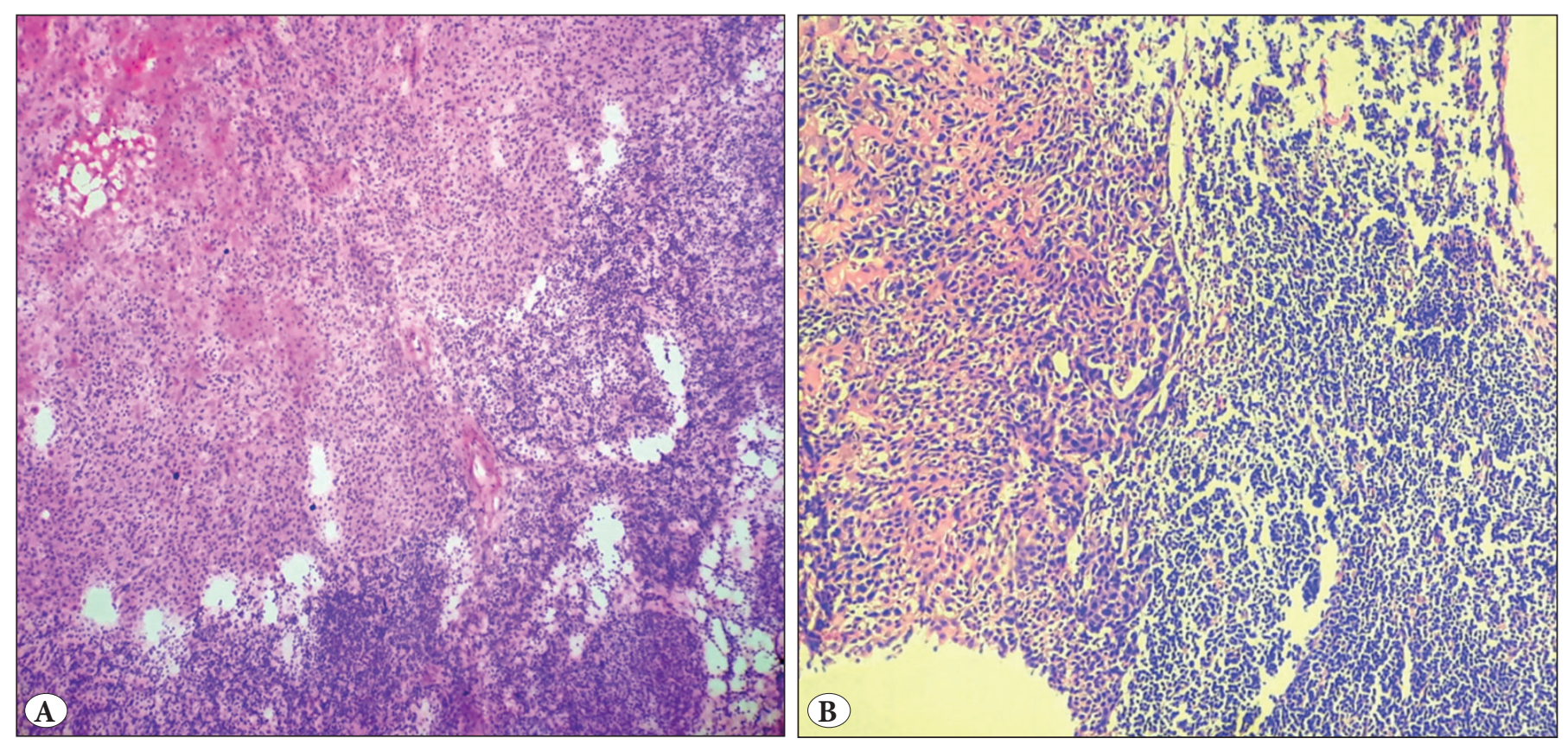

Figure 2: A) Frozen section of lymph node metastasis of squamous cell carcinoma (H\&E; x100). B) Permanent section of lymph node metastasis of squamous cell carcinoma (H\&E; x100).
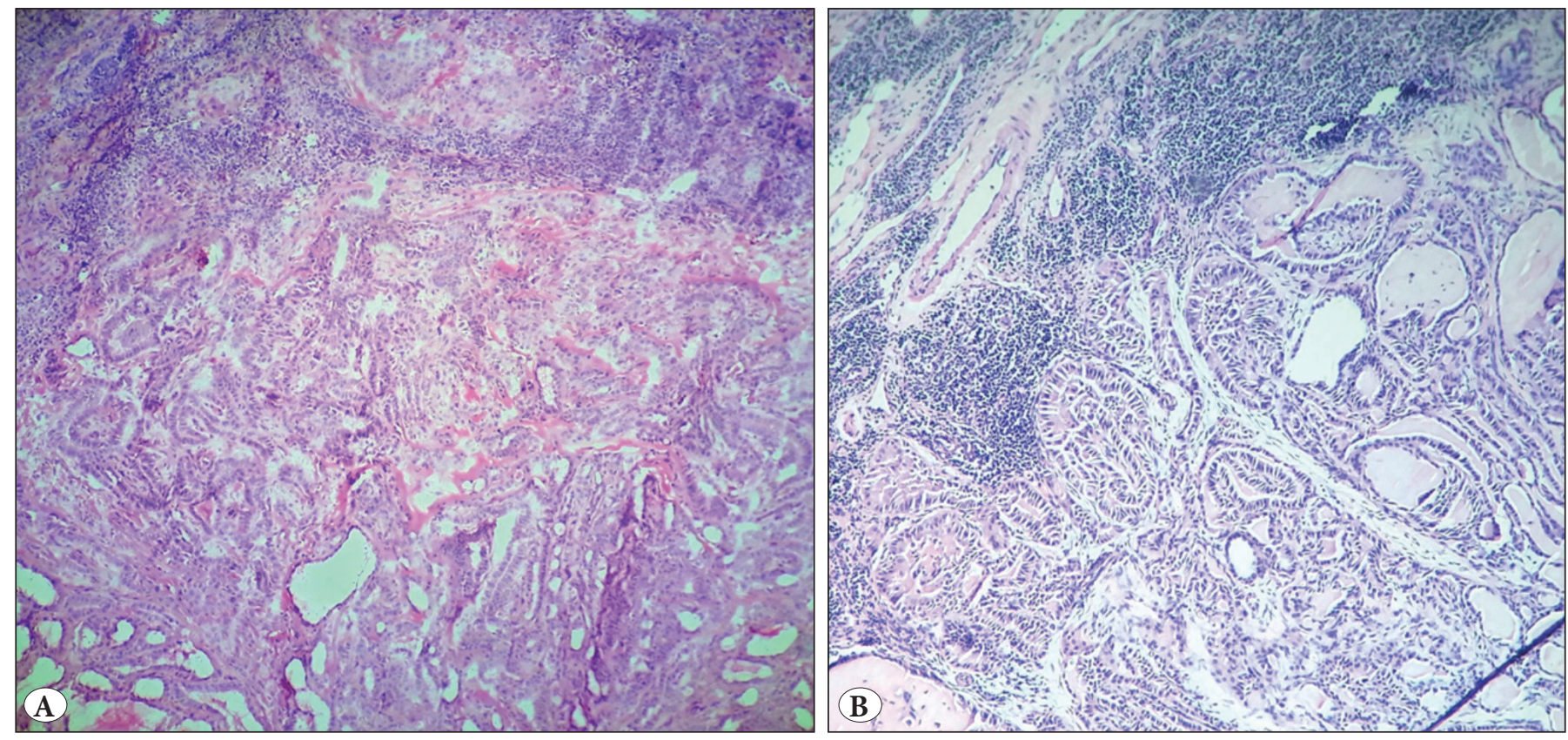

Figure 3: A) Frozen section of lymph node metastasis of thyroid papillary cell carcinoma (H\&E; x40). B) Permanent section of lymph node metastasis of thyroid papillary cell carcinoma (H\&E; $\mathrm{x} 40)$. 
Only two out of 265 cases (one carcinoma of the buccal mucosa and the other carcinoma of the tongue) showed disparity due to lack of deeper sections. These formed $0.75 \%$ of the cases. Hence, it might not be wiser to conduct an increased number of deeper section analysis and expect the overall disparity figures to reduce significantly. The time and human resource factors for doing so should also be factored in here. The researchers who have studied this particular aspect are of the opinion that the cost implications can be argued in either direction (10). Results of other studies have also shown very low rate of disparity due to lack of deeper sections when compared with the overall cases $(6,10,15)$. In our opinion, considering the time and cost factor, a lower priority can be assigned to this factor.

All other false negative cases ( $1.88 \%$ of the cases) were either due to staining issues, section folding, thick sections and/or lack pathologist's experience which contributed to misinterpretation of the sections. Studies have shown that frozen section is important and highly effective in avoiding re-operations $(16,17)$. In sites such as head and neck, margin clearance is crucial as recurrence can be very aggressive and difficult to treat (18). Hence, a false negative is more costly during intraoperative frozen section analysis as it will lead to recurrences and repeat surgeries in future. So, the issues that are main causes of false negatives should be addressed with more rigour. All these issues can be rectified with proper training of the technicians and pathologists involved in the frozen section evaluation.

On the other hand, all false positives (2.6\% of the total cases and $46.67 \%$ of the cases showing disparity) occurred from the issues with staining. It is important to avoid such issues from the view point of improving the accuracy of frozen sections. Caution should be exercised in maintaining a clean stain line with regular solution changes, following recommended timings, etc.

The true positive rate also deserves a discussion as this rate is $12.07 \%$. This indicates that a considerable number of patients benefited from the use of frozen section and it underscores the importance of frozen sections in head and neck oncosurgery.

Looking at the statistical figures, the sensitivity (82.05\%) and specificity (96.46\%) values closely match a similar-sized study by Du E et al. (19). Our specificity value also matches approximately with the values found in other studies (5, $11,20)$. As can be seen from the likelihood ratio analysis, frozen section indicates a high increase in the likelihood of the disease $(\mathrm{LR}+>10)$. Also, the LR- value is 0.19 , which is above 0.1 but below 0.2 . This indicates that frozen sections have been moderately effective in indicating reduction in the likelihood of the disease though the negative results cannot be considered to be conclusive (21). The above values come close to the values found in the study by Carvalho et al. (22). However, to avoid repeat surgery, it is desirable that the negative results be more conclusive. This aspect should be improved in our particular setup. Currently, false negatives due to technical faults contribute $71 \%$ of the total false negative cases. If we can reduce the technical errors by $50 \%$, it can be shown that LR- figure can be improved to 0.12 . This will be a very good improvement over the present condition. It should be kept in mind that samples were all intraoperative and it might not be correct to generalize the above statement beyond such cases.

Our overall accuracy rate was $94.4 \%$ (5.6\% discordance). Other studies have shown the accuracy rate of head and neck frozen sections to be in the range of $96 \%$ to $98 \%$ (6, 10). Intraoperative Consultation in Surgical Pathology (23), published by Cambridge University press in 2010, has put the error rates at $<2 \%$ after consolidating results from about six relevant studies. Our accuracy rate is slightly below, but not grossly off the mark.

In conclusion, the present study shows frozen section reports are reasonably accurate and essential in the head and neck oncosurgery. The operating surgeon should also be aware of the limitations of frozen section $(5,24)$. We recommend a minimum of 3-4 sections, optimum cryostat temperature, good section thickness and quality staining for a good concordance rate. The analysis and discussion presented above is mainly from the perspective of a tertiary cancer center with resource constraints that has just started frozen section analysis and we believe such analysis can be useful for centers similar to ours. As observed in other studies $(6,8,25)$, we have also experienced that periodic review of the correlation between frozen section diagnosis and permanent section diagnosis is needed for better service quality and we strongly suggest that centers, and especially those at the starting stage, follow such review process.

\section{CONFLICT of INTEREST}

The authors declare no conflict of interest. The study is not sponsored by any institution.

\section{REFERENCES}

1. Gal AA. The Centennial Anniversary of the Frozen Section Technique at the Mayo Clinic. Arch Pathol Lab Med. 2005;129:1532-5.

2. Recent Concepts In Sarcoma Treatment. Ryan J, Baker L, editors. Dordrecht: Springer Netherlands; 1988. 81-8. 
3. Sparkman RS. Reliability of frozen sections in the diagnosis of breast lesions . Ann Surg. 1962;155:924-34.

4. Haque R, Contreras R, McNicoll MP, Eckberg EC, Petitti DB. Surgical margins and survival after head and neck cancer surgery. BMC Ear Nose Throat Disord. 2006;6:2.

5. Hatami H, Mohsenifar Z, Alavi SN. The diagnostic accuracy of frozen section compared to permanent section: A single center study in Iran. Iran J Pathol. 2015;10:295-9.

6. Mahe E, Ara S, Bishara M, Kurian A, Tauqir S, Ursani N, Vasudev P, Aziz T, Ross C, Lytwyn A. Intraoperative pathology consultation: Error, cause and impact. Can J Surg. 2013;56:E13-8.

7. Wellnitz U, Binder B, Fritz P, Friedel G, Schwarzmann P. Reliability of telepathology for frozen section service. Anal Cell Pathol. 2000;21:213-22.

8. Ahmad Z, Barakzai MA, Idrees R, Bhurgri Y. Correlation of intraoperative frozen section consultation with the final diagnosis at a referral center in Karachi, Pakistan. Indian J Pathol Microbiol. 2008;51:469-73.

9. Cook JA, Jones AS, Phillips DE, Soler Lluch E. Implications of tumour in resection margins following surgical treatment of squamous cell carcinoma of the head and neck. Clin Otolaryngol Allied Sci. 1993;18:37-41.

10. Olson SM, Hussaini M, Lewis JS Jr. Frozen section analysis of margins for head and neck tumor resections: Reduction of sampling errors with a third histologic level. Mod Pathol. 2011;24:665-70.

11. Esbona K, Li Z, Wilke LG. Intraoperative imprint cytology and frozen section pathology for margin assessment in breast conservation surgery: A systematic review. Ann Surg Oncol. 2012;19:3236-45.

12. Eldeeb H, Macmillan C, Elwell C, Hammod A. The effect of the surgical margins on the outcome of patients with head and neck squamous cell carcinoma: Single institution experience. Cancer Biol Med. 2012;9:29-33.

13. David AN, Richard JZ. Interinstitutional comparison of frozen section turnaround time. Arch Pathol Lab Med. 1997;121:559-68.

14. White VA, Trotter MJ. Intraoperative consultation/final diagnosis correlation: Relationship to tissue type and pathologic process. Arch Pathol Lab Med. 2008;132:29-36.
15. Walts AE, Marchevsky AM. Root cause analysis of problems in the frozen section diagnosis of in situ, minimally invasive, and invasive adenocarcinoma of the lung. Arch Pathol Lab Med. 2012;136:1515-21.

16. Jorns JM, Visscher D, Sabel M, Breslin T, Healy P, Daignaut $\mathrm{S}$, Myers JL, Wu AJ. Intraoperative frozen section analysis of margins in breast conserving surgery significantly decreases reoperative rates: One year experience at an ambulatory surgical center. Am J Clin Pathol. 2012;138:657-69.

17. Hosseini M, Alizadeh Otaghvar HR, Tizmaghz A, Shabestanipour G, Arvaneh S. Evaluating the accuracy of fine needle aspiration and frozen section based on permanent histology in patients with follicular lesions. Med J Islam Repub Iran. 2015;29:239.

18. Jaafar H. Intra-operative frozen section consultation: Concepts, applications and limitations. Malays J Med Sci. 2006;13:4-12.

19. Du E, Ow TJ, Lo YT, Gersten A, Schiff BA, Tassler AB, Smith RV. Refining the utility and role of frozen section in head and neck squamous cell carcinoma resection. Laryngoscope. 2016;126:1768-75.

20. Khoo JJ. An audit of intraoperative frozen section in Johor. Med J Malaysia. 2004;59:50-5.

21. The Patient History. 2nd ed. Henderson MC, Tierney LM, Smetana GW, editors. New York: McGraw-Hill; 2012.30.

22. Carvalho MB, Soares JM, Rapoport A, Andrade Sobrinho J, Fava AS, Kanda JL, Lehn CN, Walder F, Menezes MB, Negri SL. Perioperative frozen section examination in parotid gland tumors. Sao Paulo Med J. 1999;117:233-7.

23. Intraoperative consultation in surgical pathology. Ranchod M, editor. Cambridge: Cambridge University Press; 2010

24. Kaufman Z, Lew S, Griffel B, et al. Frozen-section diagnosis in surgical pathology: A prospective analysis of 526 frozen sections. Cancer. 1986;57:377-9.

25. Raab SS, Tworek JA, Souers R, Zarbo RJ. The value of monitoring frozen section-permanent section correlation data over time. Arch Pathol Lab Med. 2006;130:337-42. 